\title{
ATP-gated P2X7 cation channel synergistically potentiates the cytotoxic effect of doxorubicin in lymphoma model (in vitro)
}

\section{Background}

Chemotherapy can activate the immune system by eliciting immunogenic cell death (ICD) of cancer cells mediated by the release of danger signals, including ATP. The purinergic P2X7 receptor is an ATP-gated cation channel expressed by hematopoietic as well as by many tumor cells (Scheuplein et al. 2009). Basal activation of P2X7 is beneficial for tumor growth, but strong stimulation of P2X7 can kill tumor cells (Roger et al. 2015). Little is known about the role of $P 2 X 7$ in the context of chemotherapy. We therefore studied the effects of $P 2 X 7$ stimulation on Doxorubicin (DOX) toxicity in lymphoma cell lines

Objective
To evaluate $P 2 X 7$ as a possible therapeutic target to

To evaluate $P 2 X 7$ as a
improve cancer therapy.

\section{Materials \& Methods}

Cell lines: Yac-1 and A20 (mouse T- and B cell lymphoma lines, respectively). Drugs: ATP, doxorubicin (DOX), and Bortezomib (BTZ).

Cell death and pore formation were monitored by DAPI uptake and the acid phosphatase cell death assay. Pre-apoptotic phosphatidyl-serine exposure and translocation of Calreticulin to the cell surface were monitored by flow cytometry.

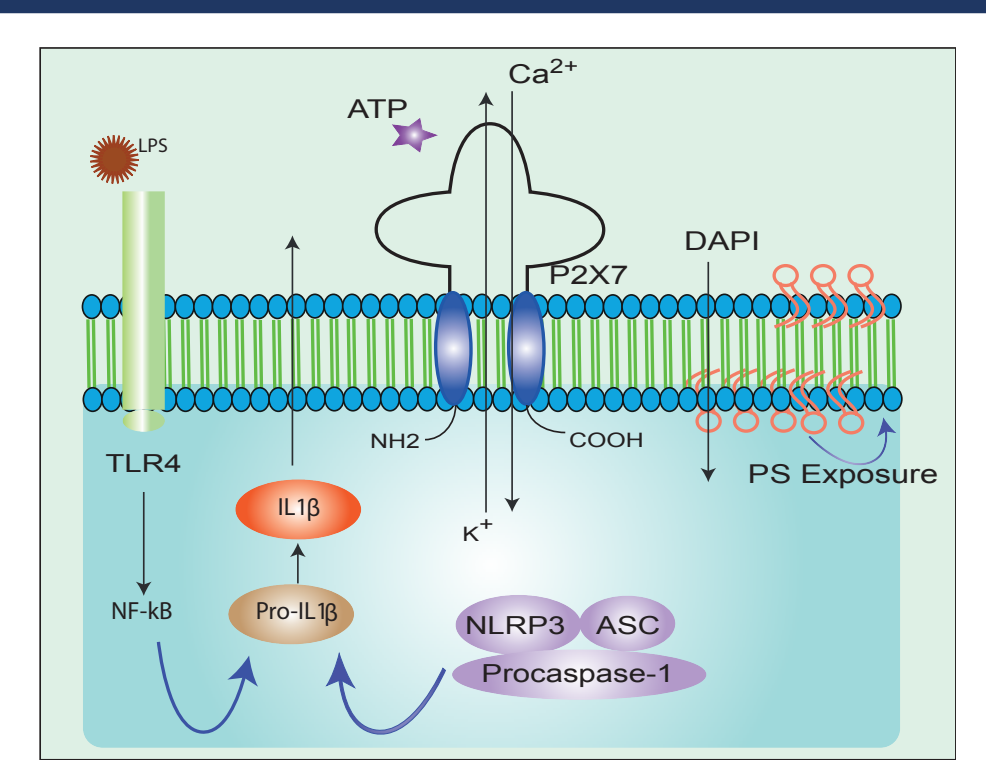

Results

Fig. 2) Gating of P2X7 increases the uptake of DOX and synergistically
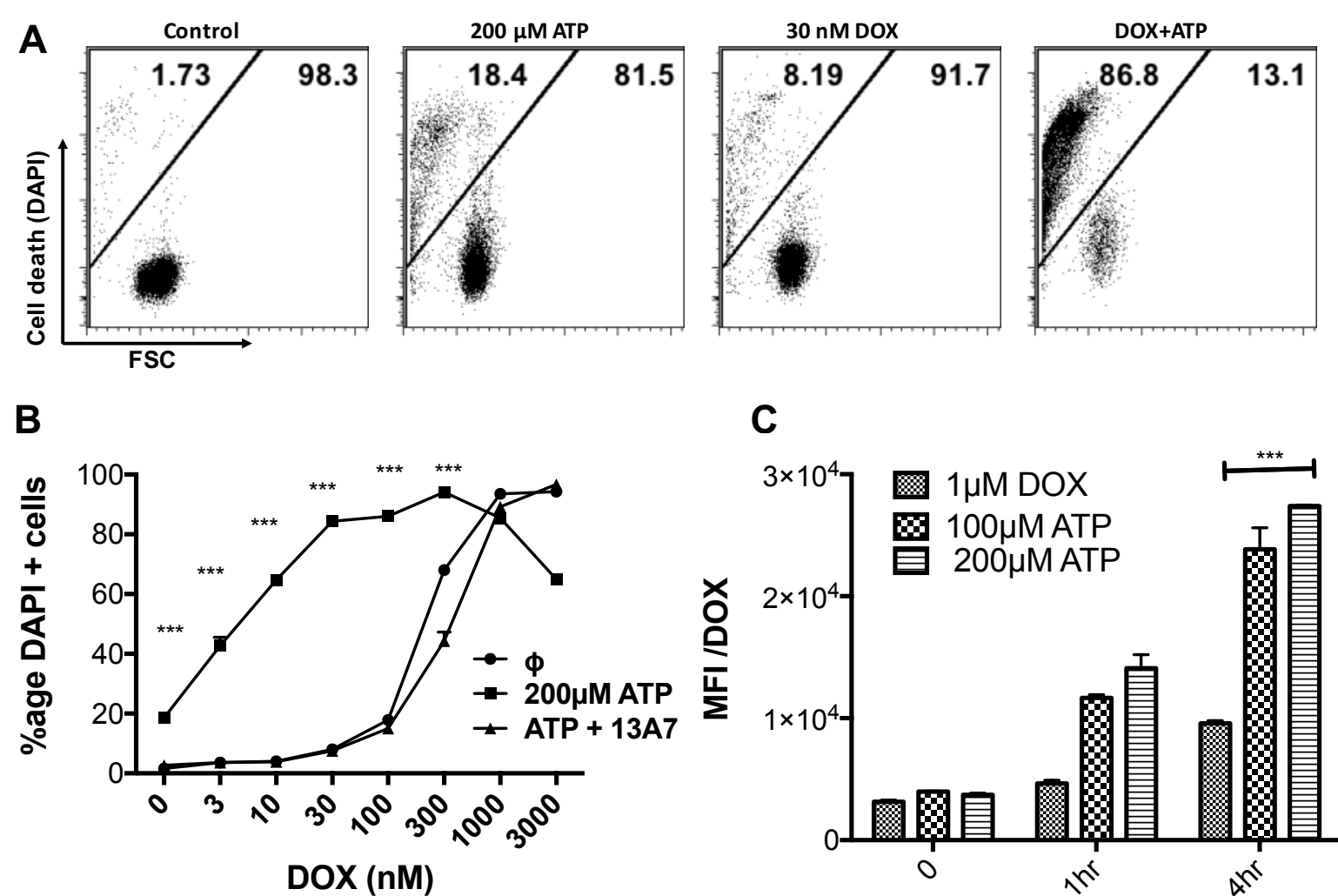
Yac-1 cells were incubated overnight with the indicated concentrations of DOX and ATP, and
stained with DAPI to indicate cell death. In B, pre-incubation with the P2X7-inhibitory nanobody 13 A7 shows that the effects of ATP are P2X7-dependent. In C, presence of ATP significantly increases the uptake of Dox.

CONTACT INFORMATION: s.j.jved@uke.de; haag@uke.de
Fig. 3) P2X7 activation augments BTZ induced cytotoxicity
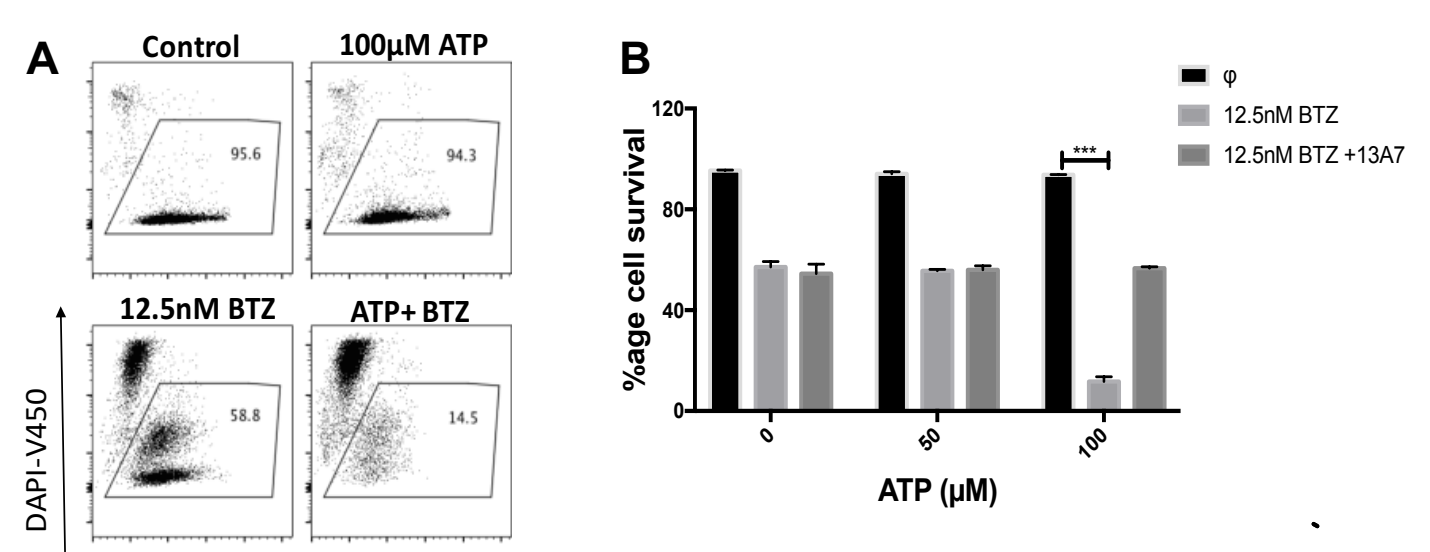

A20 cells were incubated overnight with the indicated concentrations of BTZ and ATP, and stained with DAPI to indicate cell death. In $B$, pre-incubation with the P2X inhibitory nanobody $13 A 7$ shows that the effects of ATP are P2X7-dependent. Fig. 4) P2X7 activation enhances ER stress

A
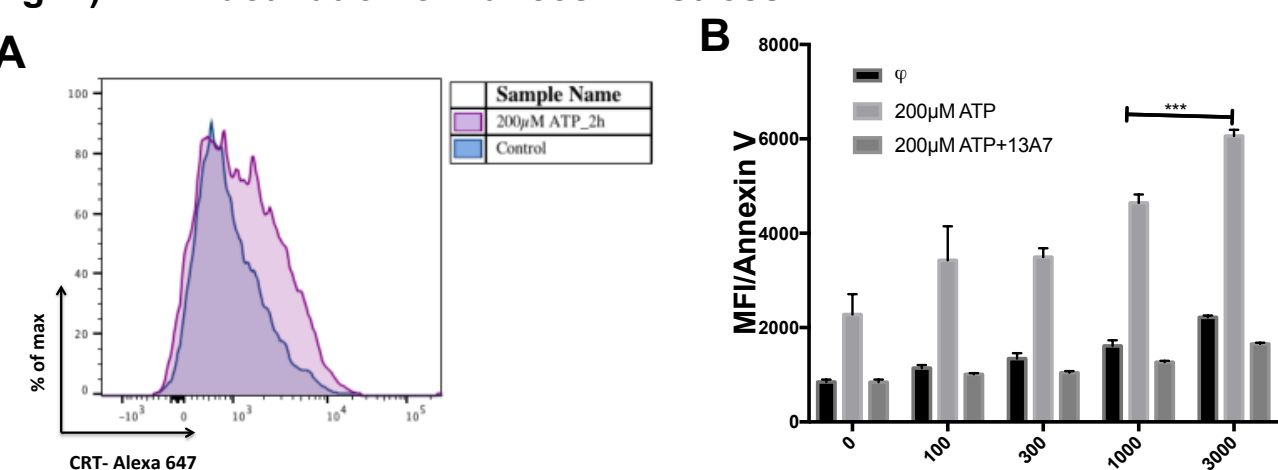

A20 cells were incubated for 2hours with $200 \mu \mathrm{M}$ ATP and stained for extracellular Calreticulin (Figure A). In B, cells were treated with indicated concentration of DOX exposure.
Summary

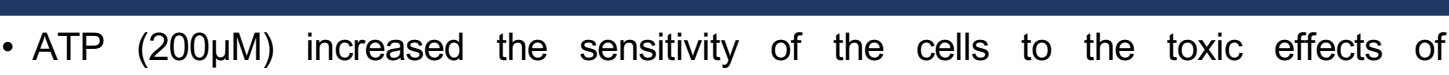
chemotherapeutic drugs i.e. DOX and BTZ (Fig. 2 \& 3). This effect was dependent on $\mathrm{P} 2 \mathrm{X7}$, since it was blocked by pre-incubating the cells with the P2X7-inhibitory nanobody $13 \mathrm{~A} 7$.

- Co-administration of ATP with DOX significantly increased DOX uptake within one hour of treatment (Fig. 2C), although no cytotoxic effects could be observed at this time point (not shown). This is likely the result of P2X7 mediated membrane pemeabilization.

- In P2X7-transduced A20 mouse lymphoma cells, ATP increased the surface translocation of Calreticulin and phosphatidyl-serine, a sign of ER stress and apoptosis. This effect was reversed by the P2X7 antagonist nanobody 13A7 (Fig. 4A \&

Conclusion

1.P2X7 synergizes the cytotoxic effects of chemotherapeutic agents to stimulate the immunogenic cell death pathway (mainly P2X7 $\mathrm{k}$ variant). The possible mechanisms for synergistic cytotoxicity are:

- Increased cell permeabilization may enhance the chemotherapeutic drugs uptake - High intracellular $\mathrm{Ca}^{2+}$ and reactive oxygen species induced ER stress, may also results in phosphorylation of elF2alpha and translocation of Calreticulin (CRT) on cell surface

2. P2X7_k expression is beneficial for host by potentiating antitumor immune response
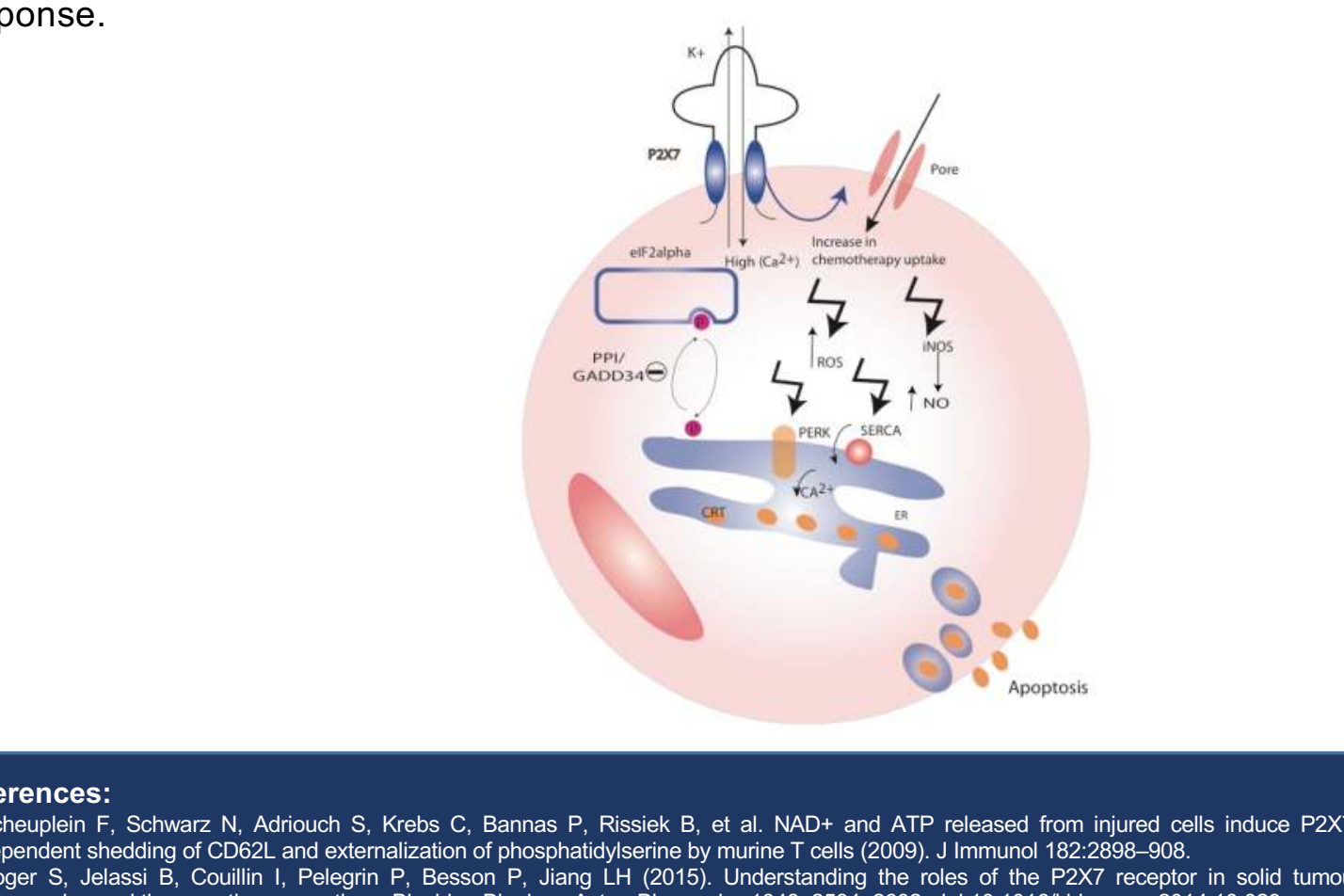